\title{
ION DYNAMICS IN MAGNETOSONIC SHOCK FRONT
}

\author{
Kichigin G.N. \\ Institute of Solar-Terrestrial Physics, \\ Irkutsk, Russia,king@iszf.irk.ru
}

\begin{abstract}
I address the ion dynamics at the front of magnetosonic shocks moving at different angles $\theta$ to the magnetic field vector. I employ a shock discontinuity model in which the ramp potential difference is taken into account. The analysis conditionally separates all the ions incoming to the front of oblique magnetosonic shocks into the following categories: 1) transient, 2) reflected, 3) gyrating in front of the ramp, 4) pickup in the ramp. Both gyrating and pickup ions are shown to be present temporarily at the magnetosonic shock front at any angles $\theta$. In the end, both the former and the latter appear to be transient in a strictly transverse magnetosonic shock; and either transient or reflected, in an oblique magnetosonic shock. I have found the critical angle $\theta^{*}$ that separates ions into transient and reflected in an oblique magnetosonic shock. The critical angle $\theta^{*}$ depends both on the velocity of the particles, incident on
\end{abstract}

\section{INTRODUCTION}

The structure of the shock front of quasiperpendicular magnetosonic shock waves (MSSW) has been studied in most detail [Leroy, 1982; Leroy et al., 1983; Balogh, Treumann, 2013]. The structure of the shock discontinuity of transverse MSSW can be represented schematically as follows: plasma upstream, a slight increase in the magnetic field - foot, a region of sharp differences in magnetic and electric fields - ramp, overshoot-undershoot oscillations of the magnetic field behind the ramp, plasma downstream. In the spatial domain called the ramp, the charge separation produces an ion-reflecting potential difference of such magnitude that it can significantly decelerate the ion flux incident on the MSSW front, whereby sharp differences are formed in ion velocity and density in the ramp. The formation of the foot is associated with ions that escape from the ramp, turn around in front of it due to the magnetic field, and then enter the ramp again. Such a turn of ion in front of the ramp can be repeated several times. As a result, the plasma density in front of the ramp increases, thus producing the foot [Woods, 1971], where the magnetic field strengthens.

The most essential element in this complex structure of the shock discontinuity of MSSW is the ramp region with jumps of the main plasma parameters: magnetic field, potential, velocity, and density. The influence of the ramp magnetic field difference on ion motion is not so essential and can be ignored in the first approximation. This is due to the fact that for the actual magnetic field strength in the ramp the Larmor radius of plasma ions moving at a velocity close to that of the plasma upstream is larger or generally much larger than the size of the ramp $d$. Sometimes the width of the potential dif- the ramp, and on dimensions of the ramp potential difference. The most important results are that I have identified the physical cause of the production of the reflected ions having a significant energy and have revealed the mechanism for their acceleration in the ramp (surfing). In the near-Earth shock ion foreshock, these very energetic ions (from tens to hundreds of $\mathrm{keV}$ ) escaping from the magnetosonic shock front at a small angle to the front plane manifest themselves in observations as so-called field-aligned beams (FABs) and form the ion foreshock boundary.

Keywords: shock, structure of magnetosonic shock front, accelerated particles.

ference is of the order of the Debye radius, i.e. it is very narrow compared to other scales typical of the shock discontinuity. Given these circumstances, existing models representing the shock discontinuity usually consider only the potential difference in the ramp. In the zeroth approximation, magnetic field variations are ignored both in the ramp and in the foot, i.e. the magnetic field within the shock discontinuity is deemed to be homogeneous.

\section{SHOCK FRONT MODEL IN USE AND BASIC EQUATIONS}

Unfortunately, there is currently no rigorous theory that deals with a self-consistent picture of particle motion in plasma of a collisionless shock wave (CSW) and represents the CSW structure in detail. There are results of laboratory experiments, numerical calculations, and measurements of CSW parameters in space plasma. It has been reliably established that some features of the CSW structure, e.g. the foot, overshoot, and undershoot, generally depend on the motion of ion component. Both plasma ions and electrons are involved in the formation of the ramp. In the absence of a rigorous theory of CSW shock discontinuity, simple hybrid models of CSW are often used in the literature. In these models, some structural elements of CSW are specified manually; then the models are employed to analyze the dynamics of only the ion plasma component. In this work, I also restrict myself to this trivial approach. Despite the simplicity, this approach provides some details of the CSW structure, which are exhibited by observations.

As examples, I will briefly discuss some commonly used models. A widespread model is the simplest shock discontinuity model, according to which some ions inci- 
dent on the ramp are reflected from it specularly [Woods, 1971; Gosling et al., 1982; Schwartz et al., 1983; Sckopke et al., 1983; Balogh, Treumann, 2013]. In this model, the motion of ions in front of the MSSW ramp is considered in the simplest approximation, which assumes the following: 1) ions are reflected from the ramp specularly; 2) reflected ions have a velocity equal to the wave velocity; 3 ) ion dynamics are analyzed in a drift approximation. This motion of ions in front of the ramp is responsible for the critical angle $\theta_{\mathrm{cr}}=45^{\circ}$ [Balogh, Treumann, 2013], which separates two different cases of ion motion in front of the ramp. In the first case $\left(\theta>\theta_{\text {cr }}\right)$, at a time point the ion enters the ramp again. Ions, thus gyrating in front of the ramp, form a foot [Woods, 1971], which is thought to be a distinctive feature of a quasi-perpendicular MSSW. In the second case $\left(\theta<\theta_{\text {cr }}\right)$, the ion, continuously drifting upstream, goes away from the ramp, so there is no reason for forming the foot, i.e. it is absent. I think that the model with mirror reflection of ions, despite its popularity, does not work because the above three assumptions used in it are not realized in practice.

Ion motion has been extensively studied for yet another model, which takes into account only the ramp magnetic field difference [Alekseyev, Kropotkin, 1970; Toptygin, 1980; Webb et al., 1983; Chiueh, 1988; Decker, 1988]. Here, in the assumption that the magnetic moment of particles is retained, it has been shown that, repeatedly crossing the ramp, ions gain energy due to drift acceleration. In the most favorable case there is an increase in the energy of these ions with respect to the initial one by about an order of magnitude (see, e.g., [Toptygin, 1980]). However, the fact that the model accounts for the ramp potential variation, which usually occupies a small part of the ramp scale, makes a radical difference. As it turns out, the potential variation has a significant effect on the dynamics of ions, in particular, on their acceleration. For example, Lever et al. [2001] have shown that, if the spatial scale of the potential difference $d_{\psi}$ is less than $c / \omega_{\mathrm{pi}}$, the increase in the ion energy due to drift acceleration becomes negligible compared to increasing energy during acceleration in the ramp electric field due to surfing acceleration [Sagdeev, 1964; Dawson, Katsouleas, 1983; Erokhin et al., 1989; Shapiro, User, 2003; Kichigin, 1992, 1995, 2001, 2009a]. According to [Heppner et al., 1978; Balikhin et al., 1995; Newbury et al., 1998; Bale et al., 2005; Bale, Mozer, 2007], for near-Earth CSW the typical value of $d_{\psi} \approx(1-5) c / \omega_{\mathrm{pe}}$, and the spatial scale of the magnetic field difference $d_{B} \approx(0.4-1) c / \omega_{\mathrm{pi}}$, i.e. $d_{\psi}<<d_{B}$. There is reason to believe that this situation is typical for CSW in space plasma, hence the following conclusion can be drawn: as a rough approximation, ions in the MSSW ramp are generally acted on by an electric field, whereas the magnetic field effect on ion dynamics in the MSSW shock discontinuity can be ignored.

Thus, the most adequate models among the simplest ones are those which account for the presence of the ramp potential difference when analyzing the ion component dynamics. The shock discontinuity model is most often discussed in the literature. In this model, a given magnetic field on the discontinuity is constant, and a manually specified potential in the ramp increases linearly, i.e. an electric field varies abruptly: within the ramp it is constant, and out of the ramp it is zero [Ohsawa, 1990; Kichigin, 1992; Lever et al., 2001; Shapiro, User, 2003]. Another model closer to the actual one assumes that an electric field in the ramp increases linearly, i.e. the potential within the ramp increases with distance according to the parabolic law [Kichigin, 1995]. This model, presented in Figure 1, is also adopted in this work.

Below I will consider in detail the ion motion in the ramp and out of it. Referring to the results obtained below, when examining the dynamics of ions moving in electromagnetic fields of the front, the ions are classified into the following categories: 1) transient ions, which cross the ramp either immediately or along a complex trajectory and never enter it again; 2) reflected ions, which after any simple or complex movements in the MSSW front appear in front of the ramp and go upstream from it; 3) gyrating ions, which gyrate in front of the ramp and form a foot; 4) pickup ions, which drift slowly within the ramp relative to it, so they are in the ramp for a long time, slowly drifting in the direction of wave motion. A region in front of the MSSW ramp will be called the foot.

A distinctive feature of the dynamics of pickup particles is that they can accelerate in the ramp up to high energies. Note that both gyrating and pickup ions exist in the MSSW front temporarily. Thus, as shown below, both the former and the latter appear to be either transient or reflected.

The reflected particle component is termed by authors of many other articles and in particular of the reviews [Balogh, Treumann, 2013; Wilson, 2016] fieldaligned beams (FAB).

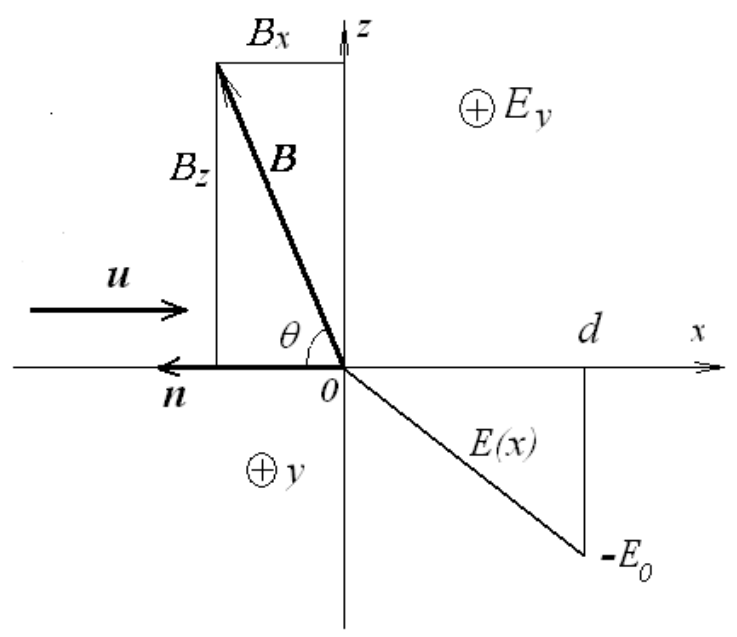

Figure 1. Shock wave structure model represented in the wave reference system. The front plane of the ramp is the YOZ plane, $\mathbf{n}$ is the normal vector to the ramp. The plasma stream falls perpendicular on the $\mathrm{YOZ}$ plane with a velocity $\mathbf{u}$. The magnetic field vector $\mathbf{B}$ is at an angle $\theta$ to the normal $\mathbf{n}$ and has $B_{x}$ and $B_{z}$ components. The potential difference is concentrated in the ramp of width $d$, and the electric field within $0 \leq x \leq d$ is directed toward the incident plasma stream and increases linearly from zero to $E_{0}$. The convective stationary electric field $E_{y}=u B_{z} / c$ is directed along the Y axis (perpendicular to the plane of the Figure) 
One of the main results of this work is the identification of physical causes of the formation of reflected particles, mechanism of their acceleration in the ramp, and trajectory of their motion in front of the MSSW ramp.

So, the purpose of this work is to examine in detail the ion dynamics in the shock front whose structure is represented by the model shown schematically in Figure 1. I think that MSSW moves in one dimension along the $\mathrm{X}$ axis in a collisionless plasma whose ions have a small velocity spread. The consideration is based on the reference system associated with the ramp. All ions incoming to the ramp will be divided into two groups. The first group contains most ions from the plasma stream incident on the ramp; the second has a minor number of ions with nearly zero mean velocity in the wave system. As we will see below, half or more of ions from the main stream immediately cross the ramp and become transient, and a small number of ions moving at approximately wave velocity can be picked up and accelerated by the electric field in the ramp. Assuming that ions from the main stream of plasma incident on the ramp are almost cold and taking into account that the number of particles in reflected, pickup, and other similar groups is small, we will roughly consider the dynamics of ions in these conventional groups in the one-particle approximation.

In the framework of this simplified shock discontinuity model in the wave system, the ion with a charge $q$ and mass $m$ in the vicinity of the front moves according to the motion equations

$$
\begin{aligned}
& d v_{x} / d t=q E_{x} / m+q v_{y} B_{z} /(m c), \\
& d v_{y} / d t=q E_{y} / m+q\left(v_{z} B_{x}-v_{x} B_{z}\right) /(m c), \\
& d v_{z} / d t=-q v_{y} B_{x} /(m c),
\end{aligned}
$$

in which the field components throughout the space

$$
B_{z}=B \sin \theta, B_{x}=-B \cos \theta, E_{y}=u B \sin \theta / c,
$$

where $c$ is the velocity of light. The $E_{x}$ component is $E_{x}=-E_{0} x / d$ in the interval $0 \leq x \leq d$ (in the ramp) and zero outside this interval (Figure 1). $E_{0}$ is related to the potential amplitude $\varphi_{\mathrm{A}}$ in the wave by $E_{0}=2 \varphi_{\mathrm{A}} / d$. We analyze the dynamics of ions in the nonrelativistic approximation, which works for the nonrelativistic MSSW of interest. To these equations add the kinetic energy equation $W=m\left(v_{x}{ }^{2}+v_{y}{ }^{2}+v_{z}{ }^{2}\right) / 2$ :

$$
d W / d t=e E_{x} v_{x}+e E_{y} v_{y} .
$$

Introducing the notation $\omega_{H}=q B / m c$, dimensionless variables $\tau=\omega_{h} t$,

$$
\begin{aligned}
& s=v_{x} / u, v=v_{y} / u, w=v_{z} / u, \chi=x \omega_{H} / u, \\
& \eta=y \omega_{H} / u, \xi=z \omega_{H} / u, \varepsilon=2 W / /\left(m u^{2}\right),
\end{aligned}
$$

and using the parameters

$$
\begin{aligned}
& R=E_{0} / B, \beta=u / c, D=R / \beta, \\
& \psi=2 e \varphi_{\mathrm{A}} /\left(m u^{2}\right), \chi_{d}=\psi / D, \Omega=D / \psi^{1 / 2},
\end{aligned}
$$

write the equations of ion motion in the ramp in a dimensionless form:

$$
\begin{aligned}
& d s / d \tau=v \sin \theta-\Omega^{2} \chi, \\
& d v / d \tau=(1-s) \sin \theta-w \cos \theta, \\
& d w / d \tau=v \cos \theta .
\end{aligned}
$$

The energy equation in the dimensionless form:

$$
d \varepsilon / d \tau=\nu \sin \theta-\Omega^{2} s \chi .
$$

Integrating this equation once over time, obtain the energy conservation law in the form of

$$
\varepsilon+\Omega^{2} \chi^{2} / 2-\eta \sin \theta=\text { const } \text {. }
$$

This ratio is used to check the numerical calculations for accuracy. The parameter $\Omega=E_{0} /\left(B \beta \psi^{1 / 2}\right)$ is associated with the presence of the electric field component $E_{x}=-$ $E_{0} x / d$ in the ramp. Since there is no $E_{x}$ out of the ramp, all the above dimensionless equations imply that $\Omega=0$ there. Equations (1)-(3) can be reduced to an equation for a single variable such as $v$ or $\chi$. As for $v$, for $\chi$ the equation has the form

$$
d^{4} \chi / d \tau^{4}+\left(1+\Omega^{2}\right) d^{2} \chi / d \tau^{2}+\Omega^{2} \cos ^{2} \theta \chi=0
$$

Here are the typical values of the parameters in use, which they possess in near-Earth MSSW. The model in hand is typical for the one-dimensional case, when a plasma stream is perpendicular to the front plane of the ramp. Assume that plasma incoming to the MSSW front with a velocity $u$ has a density $n_{0}$, and MSSW features the Alfvén Mach number $M_{\mathrm{A}}=u / V_{\mathrm{A}}$, where $V_{\mathrm{A}}=B /\left(4 \pi n_{0} m\right)^{1 / 2}$ is the Alfvén velocity in plasma; then one of the important parameters in use $D$ can be represented as follows: $D=E_{0} /(B \beta)=M_{A} \psi\left(c / \omega_{\mathrm{pi}}\right) / d$, where $\omega_{\mathrm{pi}}$ $=\left(4 \pi n_{0} e^{2} / m\right)^{1 / 2}$ is the plasma ion frequency. For the quiet solar wind $V_{\mathrm{A}}=5 \cdot 10^{6} \mathrm{~cm} / \mathrm{s}$, its velocity of incidence on near-Earth shock $u \approx 4 \cdot 10^{7} \mathrm{~cm} / \mathrm{s}$, the thermal velocity $v_{\mathrm{T}} \approx 4 \cdot 10{ }^{6} \mathrm{~cm} / \mathrm{s}$; i.e. $v_{\mathrm{T}} / u \approx 0.1<<1$; hence plasma may be considered cold. Typical values of parameters for MSSW under study: dimensionless potential $\psi \leq 1$, ramp size $d<\mathrm{c} / \omega_{\mathrm{pi}}$, Alfvén Mach number $M_{\mathrm{A}}>5$. Given the typical values of $D(D>5)$, assume in a rough approximation that $\Omega=D / \psi^{1 / 2}>>1$.

From here on, take that $\psi=1$ and, considering plasma incident on the ramp as cold, ignore transverse components of initial ion plasma velocities, i.e. set $v_{0}=w_{0}=0$.

\section{SOLUTIONS OF EQUATIONS OF ION MOTION OUTSIDE THE RAMP}

In the model adopted, in MSSW regions outside the ramp, where $\Omega=0$, equations (1) - (5) can be solved analytically. For the initial conditions $\chi=\chi_{r}, s=s_{r}, v=v_{r}$, $w=w_{r}$, the solutions have the form

$$
\begin{aligned}
& \chi=\chi_{r}+s_{r} \tau+\sin \theta\left\{\left[\left(1-s_{r}\right) \sin \theta-w_{r} \cos \theta\right](\tau-\sin \tau)+\right. \\
& \left.+v_{r}(1-\cos \tau)\right\}, \\
& s=s_{r}+\sin \theta\left\{\left[\left(1-s_{r}\right) \sin \theta-w_{r} \cos \theta\right](1-\cos \tau)+v_{r} \sin \tau\right\}, \\
& v=v_{r} \cos \tau+\left[\left(1-s_{r}\right) \sin \theta-w_{r} \cos \theta\right] \sin \tau, \\
& w=w_{r}+v_{r} \cos \theta \sin \tau+\left[\left(1-s_{r}\right) \sin \theta-w_{r} \cos \theta\right] \cos \theta(1-\cos \tau)
\end{aligned}
$$

\section{STRICTLY TRANSVERSE MSSW}

In this case, ions move in the XOY plane, so without loss of generality we can set $w=0$. As shown in [Kichigin, 1995], for a strictly perpendicular MSSW $(\theta=\pi / 2)$ equations (1)- (4) describing ion dynamics in the ramp are solved exactly. Employ these solutions to understand generally the basic laws governing the behavior of ions in the ramp whose features, as we will see below, show up in oblique MSSW. For our purposes, 
we use the solution for the coordinate and velocity of ions incident on the ramp with an initial velocity $s_{0}$ :

$$
\begin{aligned}
& \chi=\left(s_{0}-v_{\mathrm{d}}\right) \sin \left(\Omega_{1} \tau\right) / \Omega_{1}+v_{\mathrm{d}} \tau, \\
& s=\left(s_{0}-v_{\mathrm{d}}\right) \cos \left(\Omega_{1} \tau\right)+v_{\mathrm{d}}, v=\tau-\chi .
\end{aligned}
$$

Here $\Omega_{1}=\left(1+\Omega^{2}\right)^{1 / 2}, v_{\mathrm{d}}=1 / \Omega_{1}{ }^{2}$. As is clear from these formulas, a particle in the ramp in this case is moving at a constant velocity $v_{\mathrm{d}}$, on which oscillations with frequency $\Omega_{1}$ are superimposed.

The analysis of the above solutions for $\chi$ and $s$ indicates that during the first period of ion oscillation its $\chi$ coordinate is maximum at $\tau_{\mathrm{m}}$, which is determined from the relation $s=\left(s_{0}-v_{\mathrm{d}}\right) \times \cos \left(\Omega_{l} \tau_{\mathrm{m}}\right)+v_{\mathrm{d}}=0$ Supposing that $\Omega_{1} \approx D>>1, v_{\mathrm{d}}<<1, s_{0}>>v_{\mathrm{d}}$, for $\tau_{\mathrm{m}}$ obtain: $\tau_{\mathrm{m}} \approx \pi /\left(2 \Omega_{1}\right)$. If at this time point $\chi>\chi_{d}=1 / D\left(\chi_{d}=1 / D\right.$ is the dimensionless width of the ramp), the ion crosses the ramp with the velocity components $s=0, \quad v=\pi /\left(2 \Omega_{1}\right)-1 / D \approx(\pi / 2-1) / D$. As follows from (5), for such velocity values $s$ and $v$ the coordinate $\chi>0$ always; i.e., the ion that has crossed the ramp monotonically moves away from it and hence becomes transient.

For particles with velocities close to the wave velocity, $s_{0} \approx 1$, at $\tau_{\mathrm{m}}=\pi /\left(2 \Omega_{1}\right)$ the coordinate $\chi \approx s_{0} / \Omega_{1}$, and the inequality $\chi>1 / D$ takes the form $s_{0}>1$. Hence it follows that in view of the small spread in transverse velocities, approximately a half of the particles incident on the ramp cross it immediately, i.e. fall into the category of transient.

Further, by analyzing the expression for $\chi$, we see that in the first oscillation period its minimum value at $\tau$ $=3 \pi /\left(2 \Omega_{1}\right)$ :

$$
\chi_{\min }=-\left(s_{0}-v_{d}\right) / \Omega_{l}+3 \pi v_{d} d\left(2 \Omega_{1}\right) .
$$

If in this case $s_{0}<v_{\mathrm{d}}(3 \pi / 2+1), \chi_{\min }$ is positive both at this and all subsequent time points. This means that the particles that have velocities $s_{0}$, satisfying the last inequality, will never return to the foot. It is obvious that this inequality holds at very low velocities $s_{0}$ of ions, which are called ideal pickup ions. In particular, setting $s_{0} \sim v_{\mathrm{d}}$, we obtain that the ideal pickup ion, being in the ramp and oscillating with low amplitude $\sim v_{\mathrm{d}} / \Omega_{1}$, moves in it with the mean velocity $v_{\mathrm{d}}$, and hence it takes the ion $\tau_{\mathrm{d}}=\Omega$ ${ }_{1}^{2} / D \approx D$ to cross the ramp. During this time, the ion velocity component $v$ increases to $v \approx \tau_{\mathrm{d}}=D$, and the ion acquires energy $\varepsilon_{\mathrm{m}} \approx D^{2} / 2$, which is accumulated due to field acceleration $E_{y}$. The particle energy rises to such high values in the acceleration process, which is called surfing acceleration mechanism [Sagdeev, 1964; Dawson, Katsouleas, 1983; Erokhin et al., 1989; Shapiro, User, 2003; Kichigin, 1992, 1995, 2001, 2009a].

So, the analysis of solutions for the strictly perpendicular MSSW led us to the conclusion that for the cold ions incident on the ramp there may be particles gyrating in front of the ramp for a limited range of velocity components $1>s_{0}>v_{\mathrm{d}}(3 \pi / 2+1) \approx 6 v_{d}$. From $s_{0}>v_{\mathrm{d}}(3 \pi / 2+1)$ it follows that $\Omega_{1}^{2}=1+\Omega^{2}>(3 \pi / 2+1) / s_{0}$ or $D>\left[(3 \pi / 2+1) / s_{0}-1\right]^{1 / 2}$. Thus, at given $s_{0}$ the presence of gyrating particles is determined by the parameter $D$. They appear in front of the ramp only when $D>D=\left[(3 \pi / 2+1) / s_{0}-1\right]^{1 / 2}$. Given $s_{0} \approx 1, \quad D \approx(3 \pi / 2)^{1 / 2} \approx 2$. This estimate is supported by the calculations.

For ions entering the region in front of the ramp un- der the initial conditions $\chi_{0}=0, s=-s_{0}, v=v_{0}$, the solution for the ion coordinate $\chi$ takes the form

$$
\chi=\tau+\left(1+s_{0}\right) \sin \tau+v_{0}(1-\cos \tau),
$$

from which it is clear that at a time point the coordinate is bound to take a positive value. This means that any ion going out of the ramp to the foot will again returns to the ramp. As noted above, the ions that have crossed the ramp do not return. Thus, in a strictly transverse MSSW there are no reflected ions, and all the ions incident on the ramp eventually become transient.

\section{OBLIQUE MSSW}

For oblique MSSW, in the two extreme cases discussed below, for the ion motion in the ramp I managed to find an analytical solution of the system of equations (1)-(3). In general, this system can be solved only by numerical methods; and I, in particular, solved it by the Runge-Kutta method. The accuracy of the calculations according to the energy conservation law was at least $10^{-8}$.

In a particular case, namely, when describing the dynamics of particles picked up in the ramp, the solution deemed possible because for ions picked up in the ramp $s<<1$. Given this fact, in the zeroth approximation in (2) we can put $s=0$ and then system (2), (3) has the exact solution presented in [Sugihara et al., 1984; Lee et al., 1996; Kichigin, 2009a, 2009b] and has the form

$$
\begin{aligned}
& v=\operatorname{tg} \theta \sin \tau_{1}, \\
& w=\operatorname{tg} \theta\left(1-\cos \tau_{1}\right),
\end{aligned}
$$

where $\tau_{1}=\tau \cos \theta$. These solutions describe the oscillatory motion of ions in the YOZ plane with a frequency of $\cos \theta$. In the next approximation, in terms of the inequalities $s<<1, s_{0}<<1, \Omega>>1, \Omega>>\cos \theta$ adopted, substituting relations (9), (10) into (1), (2) and solving them yield relations for the coordinate $\chi$ and the velocity component $s$ :

$$
\begin{aligned}
& \chi=\left(s_{0}-\sin ^{2} \theta / \Omega^{2}\right) \sin (\Omega \tau) / \Omega+\operatorname{tg} \theta \sin \theta \sin \tau_{1} / \Omega^{2}, \\
& s=\left(s_{0}-\sin ^{2} \theta / \Omega^{2}\right) \cos (\Omega \tau)+\sin ^{2} \theta \cos \tau_{l} / \Omega^{2} .
\end{aligned}
$$

From these solutions it follows that the oscillations with a higher frequency $\Omega>>\cos \theta$ are superimposed on the ion oscillations with $\cos \theta$. The comparison of the numerical calculations with the calculations by Formula (11) for the coordinate $\chi$ shows their good agreement for $s_{0}<0.2$. Figure 2 presents the result of such a comparison for $s_{0}=0.1, D=10$.

Now turn to the analysis of (11), assuming $s_{0}>\sin ^{2} \theta / \Omega^{2}$. First, we see that the ion crosses the ramp if $\chi$ reaches the value of $1 / D$, i.e. provided that $\operatorname{tg} \theta \sin \theta / \Omega \approx 1-s_{0}$. From this relation find the critical angle $\theta^{*}$ at which ions are outside the ramp:

$$
\left.\theta^{*}=\arccos \left\{D\left(1-s_{0}\right) / 2-\left[D^{2}\left(1-s_{0}\right)^{2} / 4\right)-1\right]^{1 / 2}\right\}^{1 / 2} .
$$

If $D>>1, s_{0}<<1$, the critical angle $\theta^{*} \approx \pi / 2$. The ion that has crossed the ramp has velocity components $v_{r} \approx w_{r} \approx \operatorname{tg} \theta, s_{r}<<1$. As follows from (5)-(8), at these initial ion velocity components behind the ramp, it becomes transient.

If, when moving in the ramp, the ion does not surmount the potential difference in the ramp, then, when $\tau_{1} \geq \pi$, its coordinate becomes negative, i.e. the ion 


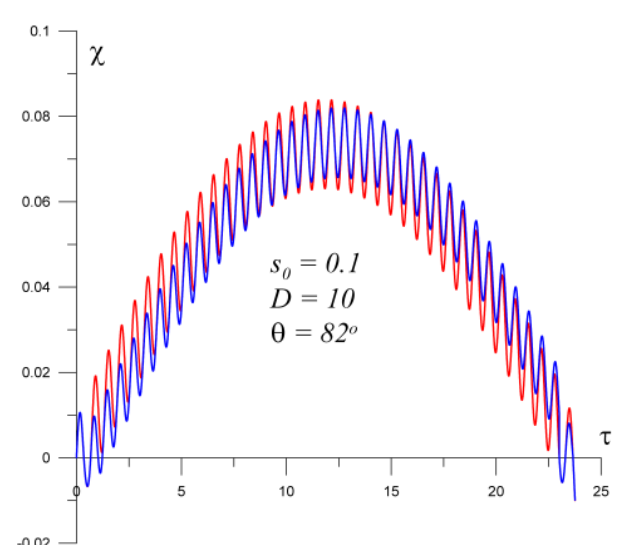

Figure 2. Coordinate as a function of time. The red curve was obtained from numerical calculations, the blue curve is the dependence obtained from Formula (11)

appears in front of the ramp, having velocity components $s_{r} \approx v_{r} \approx 0, w_{r}=2 \operatorname{tg} \theta$. The motion of ions appearing in front of the ramp with such initial velocities is governed by equations $(5)-(8)$, i.e. it is defined by the relations

$$
\begin{aligned}
& \chi \approx-\sin ^{2} \theta(\tau-\sin \tau), s \approx-\sin ^{2} \theta(1-\cos \tau), v \approx \sin \theta \sin \tau, \\
& w \approx 2 \operatorname{tg} \theta-\sin \theta \cos \theta(1-\cos \tau) .
\end{aligned}
$$

These formulas show that the ions move away from the ramp at the mean velocity $\langle s\rangle \approx-\sin ^{2} \theta$, i.e. they eventually fall into the category of reflected ions. Note that reflected ions have a maximum energy for angles $\theta$ close to $\theta^{*} \approx \pi / 2$. These energetic ions have a total velocity of $\sim 2 \operatorname{tg} \theta^{*}$ and move at an angle $\alpha$ to the YOZ plane. The angle $\alpha$ is determined from the formula $\operatorname{tg} \alpha=\langle s\rangle /\langle w\rangle \approx \sin ^{2} \theta^{*} /\left(2 \operatorname{tg} \theta^{*}\right) \approx \sin 2 \theta^{*} / 4 \approx \delta / 2$, where $\delta=$ $=\pi / 2-\theta^{*}<<1$.

Thus, the reflected ions having maximum energies move ahead of the oblique MSSW front at a very small angle $(\approx \delta / 2)$ to the $\mathrm{YOZ}$ plane. As shown in [Kichigin, 2009b], in near-Earth shock the ultimate energy of these energetic ions can reach $\sim 1 \mathrm{MeV}$. These reflected ions form a boundary of ion foreshock and move along this boundary. These very ions comprise the population of FAB (e.g., [Balogh, Treumann, 2013; Wilson, 2016] and references therein).

Referring to the calculation results, analytical solution (11) can be used for $s_{0}<0.2$. Ion trajectories for larger values of $s_{0}\left(0.2<s_{0}<1\right)$ are shown in Figure 3 . It is seen that as $s_{0}$ increases the residence time of ions in the ramp decreases and the amplitude of their spatial oscillations increases. The decrease in time leads to a decrease in the energy to which ions are accelerated in the ramp. Note also that if with low initial velocities at which ions run over the ramp $\left(s_{0}<0.2\right)$ the ions are in the ramp as pickup, with increasing $s_{0}\left(s_{0}>0.2\right)$ the ions fall into the category of gyrating ions, forming a foot in front of the ramp.

Recall that for a strictly perpendicular MSSW all the particles incident on its front, including ions perfectly picked up in the ramp, eventually cross the ramp and become transient. As we can see, in the oblique MSSW, in contrast to the strictly perpendicular one, the behavior of pickup ions in the ramp depends greatly on the angle $\theta$ whose critical value $\theta^{*}$ separates them into transient $\left(\theta>\theta^{*}\right)$ and reflected $\left(\theta<\theta^{*}\right)$. Furthermore, for the oblique MSSW the pickup ions when moving in the

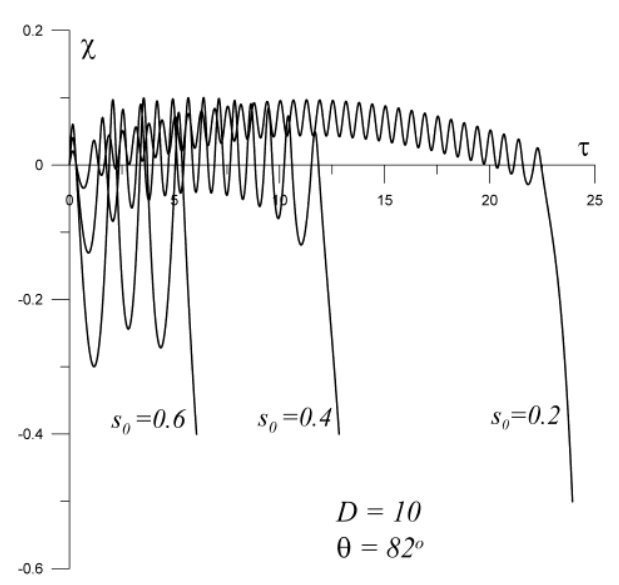

Figure 3. Ion trajectories obtained from numerical calculations for different initial velocities $s_{0}$ whose values are given near the curves. $D=10, \theta=82^{\circ}$

ramp experience a significant increase in energy as for the strictly perpendicular MSSW.

Indeed, at angles close to the critical angle, in view of (4), (5), we can see that the transient ions for the oblique MSSW behind the ramp have a kinetic energy $\varepsilon_{t} \approx \operatorname{tg}^{2} \theta^{*}$, and the reflected ones fall into the foot with an energy $\varepsilon_{\mathrm{a}} \approx 2 \operatorname{tg}^{2} \theta^{*}$. Substituting if $D>>1$ the critical angle $\cos \theta^{*} \approx 1 / D$ into the energy equation yields $\varepsilon_{t} \approx D^{2}$, $\varepsilon_{\mathrm{a}} \approx 2 D^{2}$. Notice that for the critical angles close to $\pi / 2$, which is typical for $D>1$, in the oblique MSSW the energy of transient and reflected ions is respectively two times and four times higher than the ultimate energy $\mathrm{e}_{\mathrm{m}} \approx D^{2} / 2$, with which pickup ions leave the ramp and become transient in the case of the strictly perpendicular MSSW. A similar effect of the existence of the maximum energy for reflected ions in oblique MSSW at an angle equal to the critical one is discussed in [Kichigin, 2009b].

As we have seen, in the strictly perpendicular MSSW there are no particles gyrating in front of the ramp when the parameter $D<2$. In the oblique MSSW, as derived from the calculations, the situation with gyrating ions is similar, i.e., if $D<2$, they are absent. There are however reflected particles for all $D>0$; they disappear only when $D \rightarrow 0$. To show this, turn to equations (1)(3), which for $\Omega^{2}<<1$ have an approximate analytical solution. This is the second case which has been discussed above and for which we can find an analytical solution.

As stated above, the system of equations (1)-(3) can be reduced to one equation of type (4) for $\chi$ or $v$. Solve them by the method of successive approximations, assuming $\chi=\chi_{1}+\chi_{2}, v=v_{1}+v_{2}$ and choosing the following initial conditions $s=s_{0}, \chi=v_{0}=w_{0}=0$. In the zeroth approximation, setting $\Omega=0$, obtain solutions for $\chi_{1}$ and $v_{1}$, expressed by Formulas (5), (7). Further, find the solution of interest only for $\chi$. Substituting $\chi=\chi_{1}+\chi_{2}$ in (4), where $\chi_{1}=s_{0} \tau+(1-$ $\left.s_{0}\right) \sin ^{2} \theta(\tau-\sin \tau)$, derive an equation for $\chi_{2}$ :

$d^{4} \chi_{2} / d \tau^{4}+\left(1+\Omega^{2}\right) d^{2} \chi_{2} / d \tau^{2}+\Omega^{2} \cos ^{2} \theta \cdot \chi_{2}=-\Omega^{2}(B \tau+A \sin \tau)$, where $A=\left(1-s_{0}\right) \sin ^{2} \theta, B=A+s_{0}$. Given $\Omega^{2} \ll<1$ up to terms proportional to $\Omega^{2}$, find a solution that follows from this equation: $\chi_{2}=\left(B / \Omega_{2}\right) \sin \Omega_{2} \tau-B \tau$, where $\Omega_{2}=\Omega \cos \theta$. Thus, an approximate solution of equation (4) has the form

$$
\chi \approx\left(B / \Omega_{2}\right) \sin \Omega_{2} \tau .
$$


As can be seen from this formula, if at $\tau=\pi /\left(2 \Omega_{2}\right)$ at which the coordinate is maximum $\left(\chi=\chi_{\mathrm{m}}\right)$, its value $\chi_{\mathrm{m}}$ $\geq \chi_{\mathrm{d}}=1 / D$, the particle becomes transient. If $\chi_{\mathrm{m}}<\chi_{\mathrm{d}}$, the particle returns from the ramp to the foot and moves away from the ramp as FAB. Hence, in case of the oblique MSSW moving in a cold plasma, for $\Omega^{2}<<1$ from the condition $\chi_{\mathrm{m}}=\chi_{\mathrm{d}}=1 / D$ we obtain the dependence of the critical angle $\theta^{*}$ on $D$ and $s_{0}$ :

$$
\left[\left(1-s_{0}\right) \sin ^{2} \theta *+s_{0}\right] /(\cos \theta *)+D\left(1-s_{0}\right) \sin ^{2} \theta^{*}=1 \text {. }
$$

Thus, for the oblique MSSW the critical angle, which divides particles into transient and reflected, depends both on the velocity of particles incident on the ramp $s_{0}$ and on the ramp size, which is determined by the parameter $D$. Figure 4 shows critical angles as a function of $s_{0}$ for $D=0,0.1,0.6,0.8,1,2,3,10,50$. The curve $D=0$ in Figure 4 is the relationship $\theta_{0} *=\theta_{0} *\left(s_{0}\right)=$ $=\arccos \left\{\left[\left(5-4 s_{0}\right)^{1 / 2}-1\right] /\left[2\left(1-s_{0}\right)\right]\right\}$, which is obtained by Formula (13). Curve 1 is theoretical relationship (12) $\theta^{*}\left(s_{0}\right)$ for $D=10$. As can be seen, theoretical curve 1 coincides with the calculated curve for $\theta^{*}$ at low velocities $s_{0}<0.2$, i.e. for pickup particles. It should be noted that the relationships shown in Figure 4 make it possible to estimate both the number of reflected ions and the number of transient ions for specified parameters $\theta, D$ and known ion temperature of plasma stream running over the MSSW ramp.

Note some interesting conclusions drawn from the analysis of Figure 4. The critical angle $\theta^{*}$ as a function of $D$ for ions of the main group $\left(s_{0} \approx 1\right)$ ranges from $0^{\circ}$ to $60^{\circ}$; for pickup ions $\left(s_{0} \approx 0\right)$, from $45^{\circ}$ to $90^{\circ}$. For pickup ions $\left(s_{0} \approx 0\right)$, the angle $\theta^{*}$ has a minimum value $\theta^{*} \approx 45^{\circ}$ with $D \approx 0.6$; in this case, if $D \approx 0.6$ and $\theta^{*}>45^{\circ}$, all ions incident on the MSSW ramp eventually become transient. For $D>2$ in all MSSW the critical angle $\theta^{*}$ for ions incident on the ramp with velocities $0.7<s_{0}<1$, which includes ions of the main group, is independent of $D$ and is approximately equal to $52^{\circ}$. Then, when $D>3$ for all MSSW with $\theta<52^{\circ}$, all ions incident on the ramp eventually become reflected.

Obviously, the main role in forming the structure of MSSW front is occupied by ions of the main group whose velocities are close to $s_{0} \approx 1$. According to the calculations, values of the critical angle $\theta *$, separating ions incident on the ramp into transient and reflected, essentially depend on $D$. It is therefore important to know the relationship between the critical angle and $D$ for the ions of the main group. Figure 5 shows the relationship $\theta^{*}(D)$ of interest for three values of the initial ion velocity: $s_{0}=0.9,0.95,0.99$. Referring to Figure 5 , the critical angle for ions of the main group decreases dramatically when $D$ varies within $2>D>0.2$, and if $D<$ 0.2 the angle $\theta^{*}$ is virtually independent of $D$.

\section{CONCLUSIONS}

From the MSSW shock discontinuity model employed in this paper, which accounts for the ramp potential difference, it follows that all ions incident on the ramp of oblique MSSW can be conventionally classified into the following categories: 1) transient ions that immediately cross the ramp and never come back to it again;

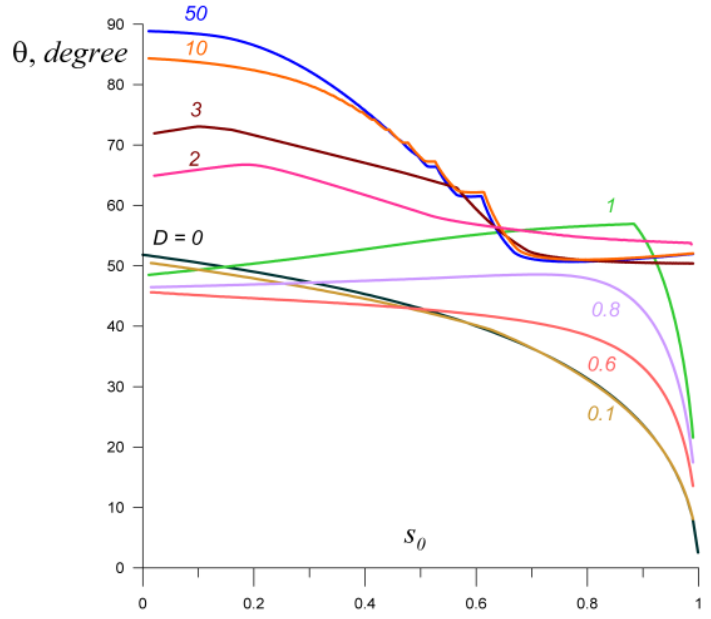

Figure 4. Critical angle $\theta^{*}$ as a function of $s_{0}$ for $D=0,0.1$, $0.6,0.8,1,2,3,10,50$

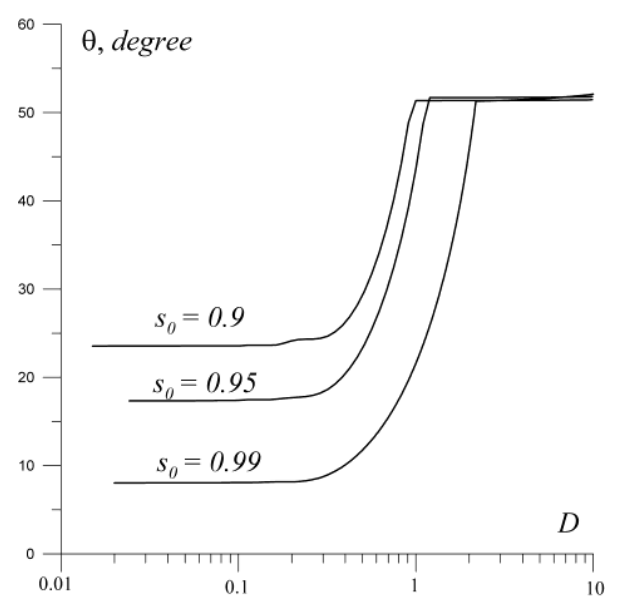

Figure 5. Critical angle $\theta^{*}$ as a function of $D$ for $s_{0}=0.9$, $0.95,0.99$

2) reflected ions that after simple or complex movements in the ramp go out of it and move upstream from the ramp; 3) gyrating ions that form a foot in front of the ramp; 4) pickup ions that within the ramp slowly drift relative to it, thus staying in the ramp for a long time. Both gyrating and pickup ions are present at the magnetosonic shock front temporarily at any angles $\theta$. In the end, both the former and the latter appear to be transient in a strictly transverse magnetosonic shock; and either transient or reflected, in an oblique magnetosonic shock.

In a strictly transverse magnetosonic shock, a small number of pickup ions, which eventually leave the ramp and become transient, are accelerated in the ramp up to energies of order of $D^{2} m u^{2} / 2$; and pickup particles in oblique magnetosonic shocks, to energies up to $D^{2} m u^{2}$.

It has been shown that in oblique MSSW a small number of ions due to the low initial velocity, when turning around in the ramp, temporarily become pickup. These ions due to surfing acceleration in the ramp acquire an energy up to $2 D^{2} m u^{2}$. With this energy, the ions go out of the ramp and then move upstream, forming a population of reflected particles. Thus, the physical cause of forming reflected ions having a high energy and the mechanism of their acceleration in the ramp have been identified. As follows from [Kichigin, 2009 b], in the near-Earth shock the ultimate energy 
$2 D^{2} m u^{2}$ of these energetic ions may be as high as 1 $\mathrm{MeV}$. These reflected ions form a boundary of ion foreshock and move along this boundary. These very ions represent the population of field-aligned beams.

The critical angle $\theta^{*}$ has been found which divides the plane $\theta s_{0}$, on which there may be oblique MSSW, into two regions (Figure 4). In one of the regions (with $\left.90^{\circ} \geq \theta>\theta^{*}\right)$, all ions incident on the MSSW ramp become transient; and in another one (with $0<\theta<\theta^{*}$ ), reflected. The critical angle $\theta^{*}$ separating ions incident on the ramp into transient and reflected depends both on the velocity of the particles incident on the ramp $s_{0}$ and on the spatial scale of the ramp, which is determined by the parameter $D$. For large values of $D(D>>1$, the ramp size $d<<c / \omega_{\mathrm{pi}}$ ) for most ions incoming to the ramp, which have a velocity close to the wave velocity $\left(s_{0} \approx 1\right)$, the critical angle $\theta * \approx 52^{\circ}$. With decreasing parameter $D$, the critical angle $\theta^{*}$ for most ions $\left(s_{0} \approx 1\right)$ decreases and tends to zero when $D \rightarrow 0$.

It has been established that the foot is present in all MSSW if $D>2$ and absent if $D<2$. The presence or absence of the foot does not depend on $\theta^{*}$.

Special appreciation is owed to Eselevich V.G. for his interest in this work and useful discussions. The work was performed with budgetary funding of Fundamental Research Program of the RAS Presidium No. 23 "HighEnergy Physics and Neutrino Astrophysics" under the project "Cosmic Rays in Heliospheric Processes as inferred from Ground and Stratospheric Observations".

\section{REFERENCES}

Alekseyev I.I., Kropotkin A.P. Passage of energetic particles through a magnetohydrodynamic discontinuity surface. Geomagnetism and Aeronomy. 1970, vol. 10, p. 755.

Bale S.D., Mozer F.S. Measurement of large parallel and perpendicular electric fields on electron spatial scales in the terrestrial bow shock. Phys. Rev. Lett. 2007, vol. 98, iss. 20, id. 205001. DOI: 10.1103/PhysRevLett.98.205001.

Bale S.D., Balikhin M.A., Horbury T.S., Krasnoselskikh V.V., Kucharek H., Mobius E., Walker S.N., Balogh A., Burgess D., Lembege B., Lucek E.A., Scholer M., Schwartz S.J., Thomsen M.F. Quasi-perpendicular shock structure and processes. Space Sci. Rev. 2005, vol. 118, pp. 161-203. DOI 10.1007/s11214-005-3827-0.

Balikhin M., Gedalin M., Krasnosselskikh V. The scales in quasiperpendicular shocks. Adv. Space Res. 1995, vol. 15 , pp. 247-260.

Balogh A., Treumann R.A. Physics of Collisionless Shocks. New York, Springer Science Business Media, 2013, 512 p. DOI: 10.1007/978-1-4614-6099-2.

Chiueh T. Multiple-encounter shock-drift acceleration in nearly perpendicular shocks. Astrophysical J. Pt. 1. 1988, vol. 333, pp. 366-385.

Dawson J.M., Katsouleas T. Unlimited electron acceleration in laser-driven plasma waves. Phys. Rev. Lett. 1983, vol. 51, pp. 392-396.

Decker R.B. Computer modeling of test particle acceleration at oblique shocks. Space Sci. Rev. 1988, vol. 48, pp. 195-262.

Erokhin N.S., Moiseev S.S., Sagdeev R.Z. Relativistic surfing in nonuniform plasma and generation of cosmic rays. Sov. Astronomy Lett. 1989, vol. 15, no. 1, pp. 3-6.

Gosling J.T., Thomsen M.F., Bame S.J., Feldman W.C. Evidence for specularly reflected ions upstream from the quasi-parallel bow shock. Geophys. Res. Lett. 1982, vol. 9, pp. 1333-1336. DOI: 10.1029/GL009i012p01333.
Heppner J.P., Maynard N.C., Aggson T.L. Early results from ISEE-1 electric field measurements. Space Sci. Rev. 1978, vol. 22, pp. 777-789.

Kichigin G.N. Investigation of the ion acceleration process at a front of magnetoacoustic wave with an isomagnetic discontinuity. Sov. Phys. JETP. 1992, vol. 74, pp. 793-814.

Kichigin G.N. Properties of surfatron acceleration of electrons. JETP. 1995, vol. 81, no. 4, pp. 736-744.

Kichigin, G. N. Surfatron mechanism of acceleration of cosmic rays in galactic plasma. JETP. 2001, vol. 92, no. 6, pp. 895-903.

Kichigin G.N. Surfing and generation of cosmic rays in relativistic shock waves. 2009a, JETP, vol. 109, no. 3, pp. 408-417.

Kichigin G.N. On an origin of energetic particles in the foreshock region of the Earth's bow shock. Astronomy Lett. 2009b, vol. 35, no. 4, pp. 261-269. (In Russian).

Lee M.A., Shapiro V.D., Sagdeev R.Z. Pickup ion energization by shock surfing. J. Geophys. Res. 1996, vol. 101A, pp. 4777-4789.

Lever E.L., Quest K.B., Shapiro V.D. Shock surfing vs shock drift acceleration. Geophys. Res. Lett. 2001, vol. 28, pp. 1367-1370.

Leroy M.M. Structure of perpendicular shocks in collisionless plasma. Phys. Fluids. 1982, vol. 26, pp. 2742-2753.

Leroy M.M., Winske D., Goodrich C.C. Wu C.S., Papadopoulos K. The structure of perpendicular bow shocks. J. Geophys. Res. 1983, vol. 87, pp. 5081-5094.

Newbury J.A., Russell C.T., Gedalin M. The ramp widths of high-Mach-number, quasi-perpendicular collisionless shocks. J. Geophys. Res. 1998, vol. 103, iss. A12, pp. 2958129594.

Ohsawa Y. Conditions for ion reflection in a large amplitude magnetosonic wave. J. Phys. Soc. Japan. 1990, vol. 59, pp. 2782-2789.

Sagdeev R.Z. Collective processes and shock waves in rarefied plasma. Rev. Plasma Phys. New York, Consult. Bur., 1966, vol. 4, pp. 23-58.

Schwartz S., Thomsen M.F., Gosling J.T. Ions upstream of the Earth's bow shock: a theoretical comparison of alternative source populations. J. Geophys. Res. 1983, vol. 88, no. A3, pp. 2039-2047.

Sckopke N., Paschmann G., Bame S.J., Gosling J.T., Russell C.T. Evolution of ion distributions across the nearly perpendicular bow shock: specularly and non-specularly reflected ions. J. Geophys. Res. 1983, vol. 88, pp. 6121-6136. DOI: 10.1029/JA088iA08p06121.

Shapiro V.D., User D. Shock surfing acceleration. Planet. Space Sci. 2003, vol. 51, pp. 665-680.

Sugihara R.S., Takeuchi N., Sakai K., Matsumoto M. de Acceleration of charged particles by an electrostatic wave propagation obliquely to a magnetic field. Phys. Rev. Lett. 1984, vol. 52, pp. 1500-1503. DOI: 10.1103/PhysRevLett.52.1500.

Toptygin I.N. Acceleration of particles by shocks in a cosmic plasma. Space Sci. Rev. 1980, vol. 26, pp. 157-213.

Webb G.M., Axford W.I., Terasawa T. On the drift mechanism for energetic charged particles at shocks. Astrophys. $J$. 1983, vol. 270, pp. 537-553.

Wilson III L.B. Low frequency waves at and upstream of collisionless shocks. Low frequency waves in space plasmas. (GMS-216)-AGU-2016, pp. 269-292.

Woods L.C. On double structured, perpendicular, magnetoplasma shock waves. Plasma Phys. 1971, vol. 13, pp. 289-302. DOI: 10.1088/0032-1028/13/4/302.

How to cite this article

Kichigin G.N. Ion dynamics in magnetosonic shock front. SolarTerrestrial Physics. 2018. Vol. 4. Iss. 4. P. 19-25. DOI: 10.12737/stp44201803. 